\title{
Adverse drug reaction detection via a multihop self-attention mechanism
}

\author{
Tongxuan Zhang, Hongfei Lin* (D), Yuqi Ren, Liang Yang, Bo Xu, Zhihao Yang, Jian Wang and Yijia Zhang
}

\begin{abstract}
Background: The adverse reactions that are caused by drugs are potentially life-threatening problems. Comprehensive knowledge of adverse drug reactions (ADRs) can reduce their detrimental impacts on patients. Detecting ADRs through clinical trials takes a large number of experiments and a long period of time. With the growing amount of unstructured textual data, such as biomedical literature and electronic records, detecting ADRs in the available unstructured data has important implications for ADR research. Most of the neural network-based methods typically focus on the simple semantic information of sentence sequences; however, the relationship of the two entities depends on more complex semantic information.

Methods: In this paper, we propose multihop self-attention mechanism (MSAM) model that aims to learn the multi-aspect semantic information for the ADR detection task. first, the contextual information of the sentence is captured by using the bidirectional long short-term memory (Bi-LSTM) model. Then, via applying the multiple steps of an attention mechanism, multiple semantic representations of a sentence are generated. Each attention step obtains a different attention distribution focusing on the different segments of the sentence. Meanwhile, our model locates and enhances various keywords from the multiple representations of a sentence.
\end{abstract}

Results: Our model was evaluated by using two ADR corpora. It is shown that the method has a stable generalization ability. Via extensive experiments, our model achieved F-measure of $0.853,0.799$ and 0.851 for ADR detection for TwiMed-PubMed, TwiMed-Twitter, and ADE, respectively. The experimental results showed that our model significantly outperforms other compared models for ADR detection.

Conclusions: In this paper, we propose a modification of multihop self-attention mechanism (MSAM) model for an ADR detection task. The proposed method significantly improved the learning of the complex semantic information of sentences.

Keywords: Adverse drug reactions, Multihop self-attention mechanism, Complex semantic information, Neural network

\section{Background}

With the rapid growth of the number of drug types, it is essential to determine the safety of the drugs that are used. Adverse drug reaction (ADR) is a broad term encompassing the dangerous effects that a drug may have. ADRs may occur after short-term or longterm administration, or they may be produced by a combination of two or more drugs. In a study that was concluded in 2000, it was reported that approximately 7000 deaths [1] were caused by ADRs each year.

\footnotetext{
${ }^{*}$ Correspondence: hongfeilin@dlut.edu.cn

College of Computer Science and Technology, Dalian University of

Technology, Dalian, China
}

The systematic review of a prospective observational study stated that $5.3 \%$ of all hospital admissions are associated with ADRs [2]. Thorough knowledge of ADRs can effectively prevent their occurrence in patients [3, 4]. Therefore, ADR detection is crucial for pharmacovigilance. Data that have been previously used in ADR research came from the Federal Drug Administration's Adverse Event Reporting System (FAERS) [5, 6] and clinical electronic medical records. Because of the privacy protection, those kinds of databases are not fully open access. Moreover, those databases are updated slowly, which limits the prevention of adverse drug reactions. 
Currently, due to the exponentially growing biomedical literature and the rapid development of social media, the resources that are generated are unlimited. Due to its fascinating characteristics and great potential, automatically extracting entities and their relations from the biomedical text have attracted much research attention [7]. Our research is entirely focused on biomedical text [8] and twitter messages [9]. As shown in Fig. 1, it is an example of annotated sentences from the ADR corpora, The first sentence contains ADR, and the second sentence does not contain ADR.

In early studies, researchers used the co-occurrence method [10] to determine the existence of ADRs. If a sentence includes both a drug and adverse reactions, it suggests that those terms are probably related. However, this method ignores the genuine semantic relations between drug and adverse reactions. Some researchers used rulesbased approaches [11], but the rules are difficult to cover all situations. In recent years, researchers developed many systems for automatically extracting relations from biomedical text, such as protein-protein interactions and gene-disease relations [12, 13]. Meanwhile, some studies employed traditional machine-learning techniques in ADR detection [14, 15]. Bian et al. [16] built support vector machine (SVM) classifiers to analyze the potential adverse events. Liu et al. [17] developed a feature-based approach for the feature selection for adverse drug events (ADEs). However, biomedical relation detection based on traditional machine-learning heavily relies on feature engineering, which is a cumbersome process.

Recently, deep learning has attracted significant attention in natural language processing (NLP) due to its numerous advantages $[18,19]$, such as less feature engineering, better performances and strong representations of data compared to other systems [20]. The convolutional neural network $(\mathrm{CNN})$ and recurrent neural network (RNN) are two widely used neural network structures in biomedical relation detection. Lee et al. [21] build several semi-supervised CNN models for ADE classification. Zeng et al. [22] proposed a piece-wise CNN
(PCNN) method to automatically learn sentence-level features and select one valid instance for the relation classification. Li et al. [23] used Bi-LSTM to represent the sentence vector combining the contextual information. It was found that the CNN model could reduce the number of model parameters through local connections and parameter sharing. It could better extract local features from short sentences. The RNN model is designed to deal with long-distance sequences and is good at dealing with long-distance features. However, the contribution of each element in the sentence is the same. Meanwhile, there is no more prominent part of the sentence that determines the category of the ADR.

The segments with a stronger focus in the sentence are treated as more important, which would influence the sentence representation. Alimova et al. [24] investigated the applicability of the interactive attention network (IAN) for the identification of adverse drug reactions from user reviews. Lin et al. [25] and Ji et al. [26] introduced an attention mechanism to the PCNN-based multi-instance learning (MIL) framework to select informative sentences. Zhou et al. [27] introduced a word-level attention model to the Bi-LSTM-based MIL framework and obtain sgnificant result. By focusing on the most relevant part of the detection of adverse reactions, this method has a greater impact on the vector representation of sentences. Although previous approaches have promising results in ADR task, they are limited to a single sentence representation that provides single semantic information. In fact, multiaspect information needs to be considered when understanding a sentence, which is helpful to enhancing the ADR detection performance.

In this paper, we propose a multihop self-attention mechanism (MSAM) that is related to dynamic memory networks (DMNs) [28] to deal with these problems. The contributions of our work can be summarized as follows:

- Our model is different from the previous methods that use the single vector representation of a sentence, which cannot obtain adequate information
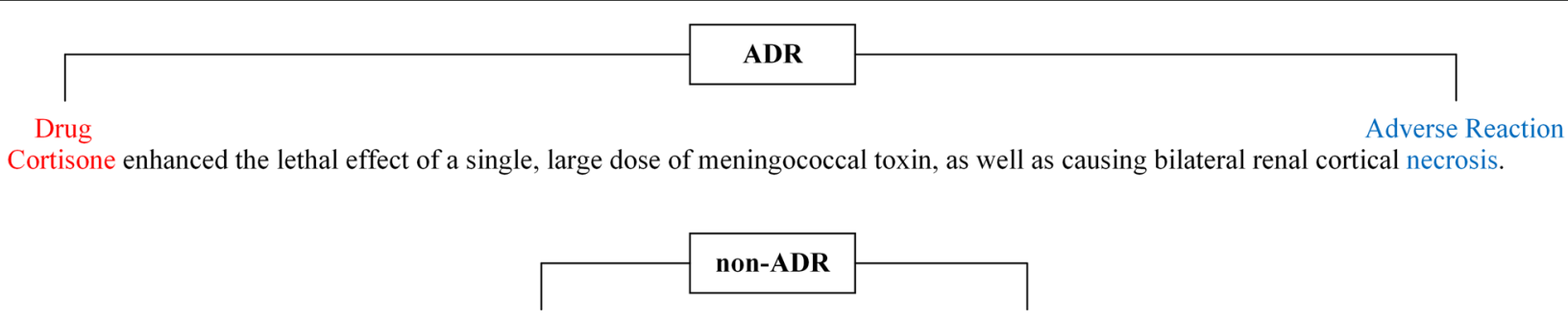

Drug Adverse Reaction

lke i'm wondering if $i$ take half a seroquel would cancel out the effects mad dizziness wellbutrin is giving me.

Fig. 1 The examples of annotated sentences in the ADR corpus 
about a sentence. Our model employs multiple vectors for the sentence representation by taking into account the previous memory results.

- By applying multiple attention mechanism, each attention step obtains different attention weights focusing on the different segments. This approach allows the MSAM to capture the different semantic information from the multiple representation of the sentence.

- Since a complete sentence contains intricate semantic information, our model applies multiple steps semantic analysis of the text to enhance the ADR classification performance. Via extensive experiments, the results show that our model achieves state-of-the-art ADR classification based on the sentence.

\section{Methods}

In this section, we explain in detail our method. First, the embedded features that are used in our neural network model are described. Second, the basic Bi-LSTM model and self-attention mechanism are introduced. At last, our MSAM model is presented. Figure 2 illustrates the MSAM that is applied to the identification of ADRs. The right side of the figure shows the details when the number of iteration steps is $\mathrm{K}=2$.

The architecture of our model consists of four components: (1) The words are represented by word vector embedding and position embedding, respectively. (2) BiLSTM can be used for extracting the contextual information in the sentence. (3) The multihop self-attention mechanism can extract complex semantic information. (4) The output layer realizes the sentence classification.

\section{Embedding input representation}

The input of our model is sentence sequence. Give a sentence $S=\left\{w_{1}, w_{2}, \ldots, w_{n}\right\}$ denote the sentence sequence. In this paper, word $w_{i}$ in the sentence sequence is represented by concatenating the word embedding and position embedding.

\section{Word embedding}

Word2Vec [29] learns low-dimensional continuous vector representations for words, which could solve the memory overflow problems that are caused by the one-hot encoding to represent the word vectors. Meanwhile, this approach could also capture the semantic information underlying the words. In recent years, word embedding has been successively applied in NLP tasks, such as sequence labeling [15], sentiment analysis [30], information retrieval [31], text classification [32] and so on. In our experiments, we downloaded a total of 2,680,617 MEDLINE abstracts from the PubMed by using the query string 'drug. Then, these abstracts were used to train word embedding by using Word2Vec [29] as the pretrained word embedding. The word $w_{i}^{\text {word }}$ is encoded into a real-values vector by using pre-trained word embedding.

\section{Position embedding}

In addition to word embedding, we also exploit position embedding to extend the input representation ability. The same word in different contexts or in different positions in a sentence has different meanings [33]. However, the word embeddings do not consider this information. Therefore, we used position embedding to capture the position features by distinguishing the relative distances between each word and the entities. For example, in the sentence "We describe a case of EGE manifested as an allergy to gemfibrozil.", the relative distances from the word 'allergy' to 'EGE' and 'gemfibrozil' are 4 and -2, respectively. Then, we mapped the relative distance to a position embedding vector. For position embedding, we randomly initialize the position vector according to a standard normal distribution and updated it when training the model. Finally, we could obtain two position embeddings $w_{i}^{\text {pos } 1}$ and $w_{i}^{\text {pos } 2}$, which are the position embeddings of $w_{i}$ with respect to drug entity and adverse reaction entity, respectively. Thus, the overall word embedding representation for $w_{i}$ is $w_{i}=\left[w_{i}^{\text {word }}, w_{i}^{\text {pos } 1}, w_{i}^{\text {pos } 2}\right]$.

\section{Extract contextual information}

RNNs perform well in processing sequential data benefits since the RNNs have the advantage of limited shortterm memory. However, when analyzing long-distance sequences, RNNs will lose the previous information, and vanishing gradient problems will occur [34]. Long ShortTerm Memory (LSTM) [35] is proposed for RNNs. It designed to deal with the long-distance sequences and solving the vanishing gradient problem.

The architecture of an LSTM unit incorporates three gates: an input gate (i), a forget gate (f), and an output gate (o). The formula of the LSTM functions are given as follows in Eqs. (1)-(6):

$$
\begin{aligned}
& f_{t}=\sigma\left(W_{f} \cdot\left[h_{t-1}, w_{t}\right]\right) \\
& i_{t}=\sigma\left(W_{i} \cdot\left[h_{t-1}, w_{t}\right]\right) \\
& \widetilde{C}_{t}=\tanh \left(W_{C} \cdot\left[h_{t-1}, w_{t}\right]\right) \\
& C_{t}=f_{t} * C_{t-1}+i_{t} * \widetilde{C}_{t} \\
& o_{t}=\sigma\left(W_{o} \cdot\left[h_{t-1}, w_{t}\right]\right) \\
& h_{t}=o_{t} * \tanh \left(C_{t}\right)
\end{aligned}
$$

Where $\sigma$ and tanh are the activation function, and $\sigma$ denotes the sigmoid function with values between 0 and 1 . 


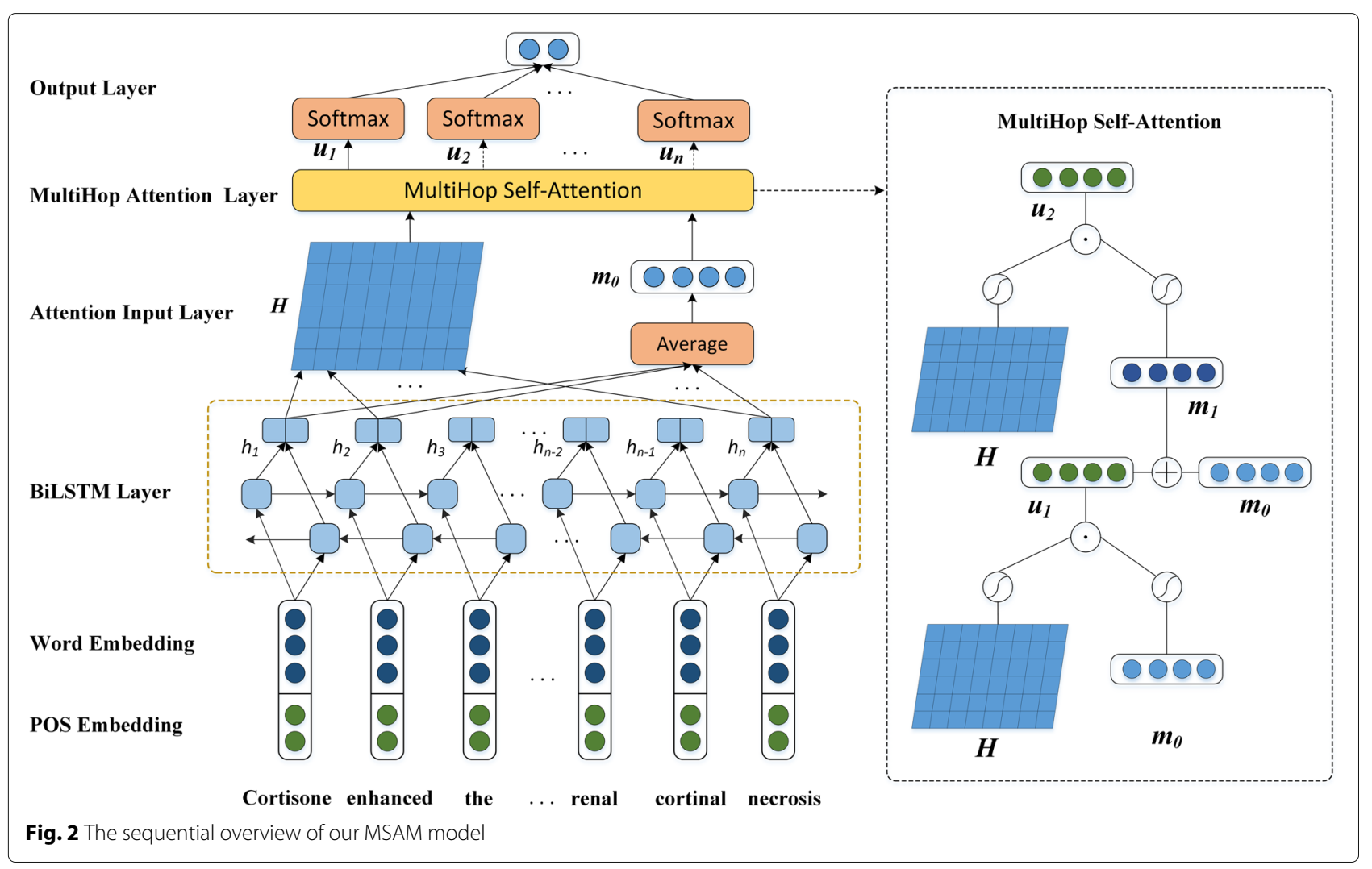

$W_{f}, W_{i}, W_{C}$, and $W_{o}$ are the weight matrices. $h_{t-1}$ represents the output of the previous cell, and $w_{t}$ represents the input of the current cell at the moment $t . C_{t}$ is the memory cell at moment $t$. All of gates are set to generate the current hidden state $h_{t}$ with the previous hidden state $h_{t-1}$ and the input token $w_{t}$.

The LSTM model is a sequential model. For the LSTM unit, it only learns the past information and cannot use future information. However, past or future information could impact the current word. Therefore, in this paper, forward and backward LSTM mechanisms were used to assess the valuable contextual information in the sentence. The Bi-LSTM could obtain each directional information in the sequences. The output $h_{t}=\left[\left(\overrightarrow{h_{t}} ; \overleftarrow{h_{t}}\right)\right]$ of Bi-LSTM is a concatenation of the forward hidden state $\overrightarrow{h_{t}}$ and the backward hidden state $\overleftarrow{h_{t}}$ at time step $t$. The generated new vector $H=\left(h_{1}, h_{2}, \ldots, h_{n}\right)$ reflects the more expressive high-level semantic meaning of the sentence. The output of the Bi-LSTM layer is a sequence of hidden vectors $H \in R^{n \times 2 d}$ where $n$ is the sequence length and $d$ is the dimensional size of the LSTM.

Language descriptions are non-standard and different. Therefore, it is especially important to find the most relevant parts of ADRs. Bi-LSTM could obtain the word dependence within the sentence and capture the internal structure of the sentence. It combines local information at a higher level through local perception. For implementation convenience, the model expects fixed-length inputs for batch processing. It is necessary to standardize the number of tokens in each sentence. In this paper, we set all sentence to be the same length by trimming longer sentences and padding shorter sentences with zero tokens. Then, we input sentence vector representation into the multihop self-attention mechanism after passing them through the Bi-LSTM layer.

\section{Self-attention mechanism}

The importances of words in a sentence are different for the ADR detection task. However, each input word shares the same weight in the input layer of neural networks. It is necessary to assign the weight for each word according to its contribution to ADR detection. The attention mechanism was first proposed in the field of visual images [36]. Since the attention mechanism is effective in machine translation [37], many researchers have applied it to NLP. The self-attention mechanism can automatically learn the weight of each word. However, a single layer of a self-attention mechanism can only focus on one part of the sentence and ignore other key parts. Therefore, we use a multiple vectors representation that focuses on different parts of the sentence to form its overall semantic representation. 


\section{Multihop self-attention mechanism}

The first multihop attention networks were proposed in the field of machine understanding and question answering [28, 38-40]. Different parts of an answer can relate to different aspects of a question [38]. The experimental results on question answering show that multihop attention networks can achieve better performance than others. Inspired by the above ideas, our model uses multihop self-attention to improve the effectiveness of ADR tasks. The iterative nature of this multihop thinking allows it to focus on different inputs during each pass so that it can explore the intricate relationship.

In many sentences, the semantic relations between drugs and adverse reactions are various. Different parts of a sentence play different roles in ADR detection. In this section, we introduce the MSAM to predict the parameters of MSAM layers through iterations. Here, we set a memory parameter $m$ and gradually update the memory parameters to iterative update the MSAM method.

Let $H=\left(h_{1}, h_{2}, \ldots, h_{n}\right)$ denote the hidden vectors of the sequence after passing through the Bi-LSTM layer. Here, $h_{t}$ is a concatenation of the forward hidden state $\overrightarrow{h_{t}}$ and the backward hidden state $\overleftarrow{h_{t}}$ at time step $t . n$ is the sequence length. In each step $k$, the formulas to compute weighted representation of sentence are as follows:

$$
\begin{aligned}
& S^{k}=\tanh \left(W_{h}^{k} H\right) \odot \tanh \left(W_{m}^{k} m^{k}\right) \\
& \beta^{k}=\operatorname{softmax}\left(w_{S}^{k} S^{k}\right)
\end{aligned}
$$

Where $W_{h}, W_{m}, W_{S}$ are the attentive weight matrices. $m^{k}$ is a separate memory vector for guiding the next self-attention step.

The initial memory parameter vector $m$ is defined based on the context vector $h_{t}$. In each step, the sentence is represented by a vector $m^{k}$ that specifically focuses on some aspects of a sentence. The memory parameter $m^{k}$ is recursively updated by (9):

$$
\left\{\begin{array}{l}
m^{0}=\frac{1}{N} \Sigma_{t} h_{t} \\
m^{k}=m^{k-1}+u^{k}
\end{array}\right.
$$

The vector $m^{k-1}$ is used as the input for the MSAM that is described in the previous step to extract the sentence representation $m^{k}$. We compute the $u^{k}$ weighted sums by multihopping the matrix $\beta^{k}$ and the Bi-LSTM hidden states $H$. The resulting structured sentence representation $u^{k}$ is shown in Eq. (10):

$$
u^{k}=\Sigma_{t} \beta^{k} H
$$

The sentence representation $u^{k}$ is the weighted sum after passing through the Bi-LSTM layer hidden states $H$.

Here, we calculate the classification weight by using $u^{k}$. Then, our model takes the average after softmax as the final classification result. The sentence probability of the ADR classification is computed as follows:

$$
\begin{aligned}
& P^{k}=\operatorname{softmax}\left(\operatorname{ReLU}\left(u^{k}\right)\right) \\
& P=\frac{1}{K} \Sigma_{k} P^{k}
\end{aligned}
$$

In this study, the experiments find that the best number of self-attention steps is $K=2$. In this case, each self-attention step gives a different attention distribution focusing on the different segments.

\section{Output and training}

After we obtain the sentence representation, we predict the classification of the sample by using a fully connected network. The softmax function is chosen as the activation function, and its calculation result is between 0 and 1 . The sum of these values is 1 . Then, the function takes the node with the highest probability as our prediction target. The formula of the softmax function is as follows:

$$
S_{i}=\frac{e^{i}}{\Sigma_{j} e^{j}}
$$

Where $S_{i}$ represents the $i^{\text {th }}$ output value of the softmax function. Prior to the prediction, we added a full connected layer to extract key features. The cost function of the model is the cross-entropy of the true class label $y$ defined as follows:

$$
C=-\Sigma_{i} y_{i} \ln S_{i}
$$

Where $y_{i}$ represents the real classification result. We trained the parameters by minimizing the loss function.

\section{Results}

\section{Experimental datasets and settings}

To evaluate the proposed approaches, we conduct an empirical evaluation based on two ADRs datasets: TwiMed and ADE. The two corpora have different language structures: the language in the literature is formal, but twitter language is informal with frequent misspellings and irregular grammar. Further, we briefly describe each dataset.

- TwiMed [9]. TwiMed corpus consists of two parts: TwiMed-PubMed and TwiMed-Twitter, which are the sentence that are extracted from PubMed and Twitters, respectively. This corpus contains three types of annotated entities: drugs, symptoms and diseases. In addition, it contains three types of relations between those entities: Reason-to-use, Outcome-positive, and Outcome-negative. In our experiments, both symptoms and diseases are considered to be adverse reactions.

Outcome-negative is used to denote that the drugs in 
Table 1 Summary statistics of the corpora

\begin{tabular}{llllll}
\hline Coupus & Documents & ADR & non-ADR & Max sentence length & Experimental data length \\
\hline TwiMed-Pubmed & 1000 & 264 & 983 & 137 & 75 \\
TwiMed-Twitter & 625 & 311 & 301 & 64 & 50 \\
ADE & 1644 & 6821 & 16695 & 90 & 90 \\
\hline
\end{tabular}

the sentence could cause adverse reactions. If the relationship between adverse reactions and drugs was labeled as Outcome-negative in the sentence, we marked the sentence as ADR (positive), otherwise, we annotate it as non-ADR (negative). The data division was similar to that used in Ilseyar et al. [24].

- ADE [8]. The ADE corpus is extracted from 1644 PubMed abstracts. There are 6821 sentences that contain at least one ADE (positive) and 16,695 sentences that contain no ADEs (negative), which have been divided. This corpus contains two types of annotated entities in ADE (positive) sentences: drugs and diseases. There are no annotated entities in the sentence with no ADEs (negative). Therefore, we did not annotate negative sentence in this task.

The summary statistics of corpora are presented in Table 1. As shown in this table, the ADE corpus contains significantly more annotations than TwiMed. Meanwhile, the datasets we used for the experiment included sentences in both PubMed and Twitter. Since the twitter application program interface does not allow for the sharing of actual tweet text, the published tweet data includes unique tweet ID but excludes the tweet text. Thus, it was necessary to obtain the tweet text by using web crawlers with the unique tweet ID. The original dataset contained a total of 1,000 tweets. When we reacquired the data using the IDs, only 625 (62.5\%) tweets were still publicly available. The Twitter and PubMed corpora were annotated by domain experts.

We attempt to combine different corpora to assess their classification accuracies. The annotations of the two datasets are different. First, both positive and negative data of the TwiMed corpus are annotated with entities. However, only positive data of the ADE corpus are annotated. Second, the TwiMed corpus includes twitter message data. However, the grammatical structure of twitter message is not standard, which makes it difficult to process and identify. Third, Pubmed sentences are usually longer than twitter sentences. Therefore, for the above problems, we also made corresponding adjustments in the parameter setting of our model.

In our experiments, we implemented our models using Keras and ran them on a TiTan GPU. We conducted that the average training time (seconds per sentence) of our method on the ADE, TwiMed-Pubmed, and TwiMedTwitter corpora are $0.065 \mathrm{~s} / \mathrm{sent}, 0.062 \mathrm{~s} / \mathrm{sent}$ and 0.051 s/sent, respectively. The word embedding parameters of our model are initialized using 100-dimensional pretrained word embeddings. The dimensionality of position embedding is 10 . The model parameters are optimized using the Adam optimizer with a learning rate of 0.01 . We used a maximum of 35 epochs to train the MSAM on each dataset. We set the batch sizes of the TwiMed and ADE dataset to 8 and 16, respectively. The number of hidden units for the Bi-LSTM layer is 50 when using Pubmed (and 20 for twitter). The best results are obtained when the number of self-attention steps is $K=2$.

All models were evaluated by using 10 -fold crossvalidation on the training set. We evaluate the performance of the classification techniques using the precision $(\mathrm{P})$, recall $(\mathrm{R})$ and F-score (F1), which are the major evaluation metrics for ADR detection on both corpora. The outcome F1 could quantify the overall performance by balancing the precision and recall.

Table 2 Classification results of the compared methods for the TwiMed corpus

\begin{tabular}{|c|c|c|c|c|c|c|}
\hline \multirow[t]{2}{*}{ Method } & \multicolumn{3}{|c|}{ TwiMed-PubMed } & \multicolumn{3}{|c|}{ TwiMed-Twitter } \\
\hline & $P$ & $\mathrm{R}$ & F1 & $P$ & $\mathrm{R}$ & F1 \\
\hline Feature-rich SVM [24] & 0.799 & 0.681 & $0.728 \pm 0.100$ & 0.752 & 0.810 & $0.778 \pm 0.047$ \\
\hline IAN [24] & 0.878 & 0.738 & $0.792 \pm 0.016$ & 0.836 & 0.813 & $0.824 \pm 0.042$ \\
\hline CNN-based method [42] & 0.849 & 0.831 & $0.835 \pm 0.060$ & 0.739 & 0.788 & $0.761 \pm 0.061$ \\
\hline multichannel CNN [43] & 0.861 & 0.780 & $0.816 \pm 0.072$ & 0.738 & 0.841 & $0.780 \pm 0.054$ \\
\hline Joint AB-LSTM [44] & 0.817 & 0.856 & $0.831 \pm 0.040$ & 0.701 & 0.828 & $0.754 \pm 0.072$ \\
\hline BiLSTM+MSAM+position & 0.858 & 0.852 & $0.853 \pm 0.057$ & 0.748 & 0.856 & $0.799 \pm 0.046$ \\
\hline
\end{tabular}


Table 3 Classification results of the compared methods for the ADE corpus

\begin{tabular}{llll}
\hline Method & $\mathrm{P}$ & $\mathrm{R}$ & $\mathrm{F1}$ \\
\hline Knowledge-based system [45] & 0.421 & 0.763 & 0.543 \\
Feature-rich classification [46] & - & - & 0.812 \\
Bi-LSTM-RNN [23] & 0.675 & 0.758 & 0.714 \\
CNNA [47] & 0.815 & 0.838 & 0.826 \\
C-LSTM-CNN [48] & 0.816 & 0.834 & $0.824 \pm 0.009$ \\
BiLSTM+MSAM & 0.847 & 0.855 & $0.851 \pm 0.013$ \\
\hline
\end{tabular}

\section{Experimental results}

In our experiments, we evaluated our proposed model via the ADR detection task, which is considered to be a classification task. In previous works, most relation detection methods assess models using large corpora, and the various semantic information inside the sentences is also ignored. In contrast, our MSAM model is designed to alleviate this problem using multiple self-attention mechanism. In our experiments, the two corpora and previous methods were compared.

\section{Evaluation on TwiMed}

We compare our proposed model with the latest models using the TwiMed corpus. Table 2 shows the performance comparisons of various models on the TwiMed corpus.

In the first two lines of Table 2, we assess the performance of the main model and baseline that was proposed by Alimova et al. [24] The feature-rich SVM method is based on the SVM with a linear kernel [41]. It considered a set of features. However, in our method, we can still get better results with a few features. In the second line, the method utilized an interactive attention network (IAN) [24] to learn the representations for targets and contexts. The IAN used attention mechanisms to detect the important words of the target expression and its full context. In addition, we consider the relative position between each word in the sentence and the entity.

From the third to the fifth lines of Table 2, the methods are our implementations. Liu et al. [42] and Quan et al. [43] proposed CNN-based methods for the relationship detection task. Kumar et al. [44] presented one model, the
Joint AB-LSTM based on the LSTM network. The models merge the semantic meanings to one single vector. However, our model uses multihop ideas to focus on the different segments of a sentence and obtain complex semantic information.

In the last line of Table 2, we give the experimental result of our proposed MSAM model. The results show that MSAM performs better than the baseline model in ADR classification. Compared with the IAN, our method obtains a $6.1 \%$ better F1 score on the TwiMed-PubMed corpus. Compared with Liu et al's [42] method, our method provides a $1.8 \%$ better F1 score on the TwiMedPubMed corpus. However, the performance on TwiMedTwitter is less pronounced. The reason is that the format of tweets is different from that of biomedical text, and a small amount of twitter data from only 625 tweets were still publicly available.

These experimental results suggest that our MSAM model could combine the contextual features that are extracted by Bi-LSTM. Compared with the feature-rich SVM [24] method, our method effectively reduces feature construction. We apply multiple self-attention steps to learn the representations for sentences. It can extract different important information in the sentence through each iteration. The multiple vectors that focus on different parts of the sentences could better represent the overall semantics. Therefore, the MSAM is better at capturing the complex semantic relations between drugs and adverse reactions and improving the results of the experiment.

\section{Evaluation on $A D E$}

We also compare our proposed model by using another corpus. Table 3 shows the performance comparisons of various models on the ADE corpus.

In the first five lines of Table 3, we present the performance of the main model and the baselines for the ADE corpus. Kang et al. [45] developed a knowledge-based relation detection system that could be successfully used to extract adverse drug events from biomedical text. The learning process relies on external knowledge and ignores sentence-specific information because of the utilization of a small amount of data. Due to the limitations of the manual rule setting, this method resulted in a high recall score but a low precision score. Sarker et al. [46] relied

Table 4 Performances obtained by using different attention mechanisms

\begin{tabular}{|c|c|c|c|c|c|c|c|c|c|}
\hline \multirow[t]{2}{*}{ Method } & \multicolumn{3}{|c|}{ TwiMed-PubMed } & \multicolumn{3}{|c|}{ TwiMed-Twitter } & \multicolumn{3}{|l|}{$\mathrm{ADE}$} \\
\hline & $P$ & $\mathrm{R}$ & $\mathrm{F} 1$ & $P$ & $\mathrm{R}$ & $\mathrm{F} 1$ & $P$ & $\mathrm{R}$ & F1 \\
\hline Self-Attention & 0.855 & 0.845 & 0.846 & 0.731 & 0.793 & 0.751 & 0.845 & 0.848 & 0.847 \\
\hline Multi-head Self-Attention & 0.829 & 0.850 & 0.841 & 0.767 & 0.800 & 0.784 & 0.820 & 0.851 & 0.836 \\
\hline Multihop Self-Attention & 0.858 & 0.852 & 0.853 & 0.748 & 0.856 & 0.799 & 0.847 & 0.855 & 0.851 \\
\hline
\end{tabular}


Table 5 Performance of various modules on the TwiMed corpus

\begin{tabular}{|c|c|c|c|c|c|c|}
\hline \multirow[t]{2}{*}{ Method } & \multicolumn{3}{|c|}{ TwiMed-PubMed } & \multicolumn{3}{|c|}{ TwiMed-Twitter } \\
\hline & $P$ & $\mathrm{R}$ & F1 & $P$ & $\mathrm{R}$ & $\mathrm{F} 1$ \\
\hline BiLSTM & 0.853 & 0.806 & 0.829 & 0.680 & 0.754 & 0.715 \\
\hline BiLSTM+position & 0.843 & 0.825 & 0.836 & 0.809 & 0.654 & 0.723 \\
\hline BiLSTM+Self-Attention+position & 0.855 & 0.845 & 0.846 & 0.731 & 0.793 & 0.75 \\
\hline BiLSTM+MSAM+position & 0.858 & 0.852 & 0.853 & 0.748 & 0.856 & 0.79 \\
\hline
\end{tabular}

on generating a large set of features representing the semantic properties from the text. However, our method only used the word embedding feature on the ADE corpus, and it could still obtain better performance. Li et al. [23] investigated joint models for simultaneously extracting drugs, diseases, and adverse drug events. It used a dependency parser, which we did not need. Huynh et al. [47] proposed the convolutional neural network with attention (CNNA) by adding the attention weights into convolutional neural networks. Song et al. [48] presented the Context-LSTM-CNN method for sentence classification. The method analyzed the data based on the abstract text that contained the data. The generalization of the method is reduced, which could not be well applied to the processing of short text data, such as twitter messages.

In the last line of Table 3, we give the experimental results of our proposed MSAM model. The memory parameter $m$ can record the important information of each iteration step of the multihop attention mechanism so that we can obtain multiple sources of information and comprehensively judge it. From the results, we observe that the MSAM model achieve the best results on the ADE corpus. Our results were better than others with a few features. Compared with the Context-LSTM-CNN method that achieves state-of-the-art results, our method obtains a $2.7 \%$ better F1 score.

\section{Performance with different attention}

In Table 4, we give experimental results of the different attention [49] models on TwiMed and ADE, respectively.

We can see from Table 4 that the results of the model obtained using multihop self-attention are better than those obtained by models using multi-head self-attention and self-attention. Our method allows the model to assess the information from different positions. The vector representation for each step in our model takes into account the results of the previous step. Our MSAM model can learn a better sentence representation by focusing on different aspects of the sentence, which makes the sentence-level multihop self-attention mechanism have a better chance of selecting the sentences containing ADRs. Therefore, the results of our model will be relatively better than those of others.

\section{Effect of various modules}

In Table 5, we also give experimental results of the basic model on TwiMed and ADE. The simplified models are described as follows:

- Bi-LSTM: The model is used as the baseline model. Others methods are based on this method. Forward and backward LSTM mechanisms extract the information in the sentence.

- Bi-LSTM+Self-Attention: The model integrates the self-attention mechanism based on the Bi-LSTM model.

- Bi-LSTM+Multihop Self-Attention: The model integrates the multihop self-attention mechanism based on the Bi-LSTM model. We conducts experiments for different iteration steps.

- Bi-LSTM+Multihop Self-Attention+position: The model integrates the position feature based on the Bi-LSTM+Multihop Self-Attention model.

Table 5 shows the results when we evaluate the position feature in experiments on the TwiMed corpus. Considering the position feature on the TwiMed corpus, the contextual information of each word in the sentence can be distinguished. The precision and recall of TwiMed-Twitter fluctuate quite widely bacause of the small amount of twitter data, further, social media language is highly informal, and user-expressed medical concepts are often nontechnical.

Table 6 shows the results for the ADE corpus when there is no position feature. The reason for these results is that the negative data in ADE corpus are not annotated. Therefore, we do not consider that a sentence may contain different relationships in the ADE corpus. Our method achieved a high F1 score of $85.1 \% \mathrm{~F} 1$, which is $0.4 \%$ better than that of the Bi-LSTM+Self-Attention method. The

Table 6 Performance of various modules on the ADE corpus

\begin{tabular}{llll}
\hline Method & $P$ & $R$ & $F 1$ \\
\hline BiLSTM & 0.812 & 0.822 & 0.817 \\
BiLSTM+Self-Attention & 0.847 & 0.848 & 0.847 \\
BiLSTM+MSAM & 0.847 & 0.855 & 0.851 \\
\hline
\end{tabular}


self-attention results are also very high because the ADE corpus contains simple information. Therefore, the results of our MSAM model on the ADE corpus are not substantially different from the results of the self-attention mechanism.

\section{Effect of the number of MSAM steps}

Table 7 shows the F1-measure with respect of the number of steps on the TwiMed corpus and ADE corpus. Step1, step2, and step3 represent the MSAM iteration steps. When the number of MSAM iteration steps is $K=2$, the model obtains the best performance. This effect might be due to the sentences not being particularly long and often containing two important aspects at most. Therefore, multiple steps may have significant effects on the multi-aspect information detection for long text. Table 7 also demonstrates that the performances on the different ADR corpora varied significantly with respect to the different numbers of steps.

\section{Effect of imbalance data}

We also get the result from up-sampling and downsampling that the ratio of positive and negative samples is $1: 1$. Table 8 shows the performance on the TwiMedPubMed and ADE corpora. The TwiMed-Twitter corpus does not have imbalance data, so we did not apply up-sampling or down-sampling to it. The up-sampling method copies the positive samples in the training dataset. Because of the small number of examples, increasing the positive examples could improve the experimental results to some extent. The down-sampling method removes the negative examples. Although some negative examples have been removed, which reduced the impact of noisy data, the small amount of data is the main reason why the results are not ideal.

\section{Case study}

Figure 3 depicts the heat map of a sentence from the TwiMed corpus that was subjected to MSAM. We gave examples from PubMed and Twitter that illustrate the effectiveness of our proposed model. The stronger the red color of a word in the sentence is, the larger the multihop self-attention layer weight of that word. The first sentence is the heat map result that was obtained by MSAM step 1 . The second sentence is the heat map result that was obtained by MSAM step 2 . In this example, we observe

Table 7 Effects of different number of steps and self-attention on both corpus (F1)

\begin{tabular}{llll}
\hline Method & TwiMed-PubMed & TwiMed-Twitter & ADE \\
\hline step1 & 0.831 & 0.786 & 0.819 \\
step2 & 0.853 & 0.799 & 0.851 \\
step3 & 0.820 & 0.789 & 0.820 \\
\hline
\end{tabular}

Table 8 Effects of up-sampling and down-sampling for imbalanced data

\begin{tabular}{llll}
\hline Corpus & $P$ & $R$ & $F 1$ \\
\hline TwiMed-PubMed & 0.858 & 0.852 & $0.853 \pm 0.057$ \\
TwiMed-PubMed (up) & 0.851 & 0.889 & $0.867 \pm 0.032$ \\
TwiMed-PubMed (down) & 0.862 & 0.842 & $0.849 \pm 0.033$ \\
ADE & 0.847 & 0.855 & $0.851 \pm 0.013$ \\
ADE (up) & 0.846 & 0.869 & $0.857 \pm 0.007$ \\
ADE (down) & 0.823 & 0.862 & $0.842 \pm 0.014$ \\
\hline
\end{tabular}

that our model is able to extract the complex semantic information from the sentence. We do not only focus on entities, which are drugs and reactions, but we also focus on finding words other than entities that can play important roles in the classification of relationships. The focus of each iteration step is different, which allows the sentence vector representation to be obtained from the multidimensional perspective.

\section{Conclusion}

Most of the neural network models only capture simple semantic information from the single representation of a sentence, which limits the performance of the ADR detection task. In fact, determining the relationship between drugs and adverse reactions requires complex semantic information. In this paper, we propose a multihop selfattention mechanism for the ADR detection task, which allows the model to capture multiple semantic information bits for the ADR detection task. By using the multistep attention mechanism, our model learns multiple vector representations that focus on different semantic information to detect the relationships between drugs and adverse reactions. Experimental results obtained for two different widely used corpora demonstrate that (i) our MSAM is effective at capturing the complex semantic information in a sentence; and (ii) our model is robust and suitable for different types of text. It is encouraging to see that our model achieves state-of-the-art results on ADR classification based on the sentence level.

Although our model achieved the best performance on the TwiMed-PubMed and ADE corpora, there is still room to improve. The performance on the TwiMed-Twitter corpus is relatively lower than that on TwiMed-PubMed. The reason for this discrepancy is that the number of training examples in the TwiMed-Twitter corpus is very limited compared with the TwiMed-PubMed corpus. Because of the particularity of twitter data, we have less available data. We obtain 625 sentences for the experiment. Meanwhile, the language in social media is highly informal. In future work, we will combine twitter data and biomedical 


\section{PubMed: $\mathrm{k}=1$ Cortisone enhanced the lethal effect of a single, large dose of meningococcal toxin, as well as causing bilateral renal cortical necrosis. \\ PubMed: $k=2$ \\ Cortisone enhanced the lethal effect of a single, large dose of meningococcal toxin, as well as causing bilateral renal cortical necrosis.}

Fig. 3 Attention heat map from MSAM $(k=2)$ for ADRs classification

\section{Twitter: $k=1$}

Topamax taken during pregnancy can cause birth defects - heart, lung, brain abnormalities, cleft palate, cleft lip, other serious health problem.

\section{Twitter: $\mathbf{k}=2$}

Topamax taken during pregnancy can cause birth defects heart, lung, brain abnormalities, cleft palate, cleft lip, other serious health problems literature data to train the model to solve the problem of insufficient twitter data.

\section{Abbreviations}

ADEs: Adverse drug events; ADRs: Adverse drug reactions; Bi-LSTM: Bidirectional long short-term memory; CNN: Convolutional neural network; DMNs: Dynamic memory networks; FAERS: The federal drug administration's adverse event reporting system; LSTM: Long short-term memory; MSAM: Multihop self-attention mechanism; NLP: Natural language processing; PCNN: Piece-wise convolutional neural network; RNN: Recurrent neural network; SVM: Support vector machine

\section{Acknowledgements}

Authors would like to thank the editor and all anonymous reviewers for valuable suggestions and constructive comments, Authors would also like to thank the Natural Science Foundation of China.

\section{Authors' contributions}

$T Z, H L, Y R, Z Y, J W$ and $Y Z$ carried out the biomedical natural language studies and the adverse drug reactions detection study. TZ developed the model, and wrote the first draft. All authors participated in manuscript preparation. All authors read and approved the final manuscript.

\section{Funding}

This work has been supported by the Natural Science Foundation of China (No. 61572102,61632011,61562080). The funding bodies did not play any role in the design of the study, data collection and analysis, or preparation of the manuscript.

\section{Availability of data and materials}

The datasets analysed during the current study are available in the https:// www.ncbi.nlm.nih.gov/pmc/articles/PMC5438461/bin/jmir_v3i2e24_app1.zip https://sites.google.com/site/adecorpus/home/document.

\section{Ethics approval and consent to participate}

Not applicable

\section{Consent for publication}

Not applicable

\section{Competing interests}

The authors declare that they have no competing interests.

Received: 21 February 2019 Accepted: 26 August 2019

Published online: 18 September 2019

\section{References}

1. Hazell L, Shakir SAW. Under-reporting of adverse drug reactions. Br Med J. 2012;29(5):385-96.

2. Chuenjid K, Noyce PR, Ashcroft DM. Hospital admissions associated with adverse drug reactions: a systematic review of prospective observational studies. Ann Pharmacother. 2008;42(7):1017-25.

3. Hakkarainen KM, Khadidja $H$, Staffan $H$. Percentage of patients with preventable adverse drug reactions and preventability of adverse drug reactions-a meta-analysis. PloS ONE. 2012;7(3):33236.
4. Sultana J, Cutroneo P, Trifirò G. Clinical and economic burden of adverse drug reactions. J Pharmacol Pharmacother. 2013;4(Supp|1):73-7.

5. Li H, Guo XJ, Ye XF, Jiang H, Du WM, Xu JF, Zhang XJ, He J. Adverse drug reactions of spontaneous reports in shanghai pediatric population. PloS ONE. 2014;9(2):89829.

6. Lindquist M. Vigibase, the who global icsr database system: Basic facts. Drug Inf J. 2008;42(5):409-19.

7. Wei CH, Peng Y, Leaman R, Davis AP, Mattingly CJ, Li J, Wiegers TC, Lu Z. Assessing the state of the art in biomedical relation extraction: overview of the biocreative $v$ chemical-disease relation (cdr) task. Database J Biol Databases Curation. 2016;2016. https://doi.org/10.1093/ database/baw032.

8. Gurulingappa H, Rajput AM, Roberts A, Fluck J, Hofmann-Apitius M, Toldo L. Development of a benchmark corpus to support the automatic extraction of drug-related adverse effects from medical case reports. J Biomed Inform. 2012;45(5):885-92.

9. Alvaro N, Miyao Y, Collier N. Twimed: Twitter and pubmed comparable corpus of drugs, diseases, symptoms, and their relations. Jmir Public Health Surveill. 2017;3(2):24.

10. Airola A, Pyysalo S, Björne J, Pahikkala T, Ginter F, Salakoski T. All-paths graph kernel for protein-protein interaction extraction with evaluation of cross-corpus learning. BMC Bioinformatics. 2008;9 Suppl 11(S11):2.

11. Fundel $K$, Kuffner $R$, Zimmer R. Relex - relation extraction using dependency parse trees. Bioinformatics. 2007;23(3):365-71.

12. Zweigenbaum $P$, Demnerfushman D, Yu H, Cohen KB. Frontiers of biomedical text mining: current progress. Brief Bioinform. 2007;8(5): 358-75.

13. Cohen AM, Hersh WR. A survey of current work in biomedical text mining. Brief Bioinform. 2005;6(1):57-71.

14. Xu J, Wu Y, Zhang Y, Wang J, Lee HJ, Xu H. Cd-rest: a system for extracting chemical-induced disease relation in literature. Database. 2016;2016:036.

15. Nikfarjam A, Sarker A, O'Connor K, Ginn R, Gonzalez G. Pharmacovigilance from social media: mining adverse drug reaction mentions using sequence labeling with word embedding cluster features. J Am Med Inform Assoc. 2015;22(3):671-81.

16. Bian J, Topaloglu U, Yu F. Towards large-scale twitter mining for drug-related adverse events. Proceedings of the 2012 international workshop on Smart health and wellbeing. Hawaii: ACM; 2012. p. 25-32.

17. Liu J, Zhao S, Zhang X. An ensemble method for extracting adverse drug events from social media. Artif Intell Med. 2016;70(9):62-76.

18. Zeng $W$, Lin $Y$, Liu Z, Sun M. Incorporating relation paths in neural relation extraction. Empirical Methods in Natural Language Processing. Copenhagen: ACL; 2017. p. 1768-77.

19. Zhang Y, Zheng W, Lin H, Wang J, Yang Z, Dumontier M. Drug-drug interaction extraction via hierarchical mns on sequence and shortest dependency paths. Bioinformatics. 2017;34(5):828-35.

20. Rajkomar A, Oren E, Kai C, Dai AM, Hajaj N, Hardt M, Liu PJ, Liu X, Marcus J, Sun M. Scalable and accurate deep learning with electronic health records. NPJ Digital Medicine. 2018;1 (1):18.

21. Lee K, Qadir A, Hasan SA, Datla V, Prakash A, Liu J, Farri O. Adverse drug event detection in tweets with semi-supervised convolutional neural networks. International Conference on World Wide Web. Perth: ACM; 2017. p. 705-14.

22. Zeng D, Liu K, Chen Y, Zhao J. Distant supervision for relation extraction via piecewise convolutional neural networks. Empirical Methods in Natural Language Processing. Lisbon: ACL; 2015. p. 1753-62. 
23. Li F, Zhang M, Fu G, Ji D. A neural joint model for entity and relation extraction from biomedical text. BMC Bioinformatics. 2017;18(1):198.

24. Alimova I, Solovyev V. Interactive attention network for adverse drug reaction classification. Conference on Artificial Intelligence and Natural Language. Cham: Springer; 2018. p. 185-96.

25. Lin Y, Shen S, Liu Z, Luan H, Sun M. Neural relation extraction with selective attention over instances. Meeting of the Association for Computational Linguistics. Berlin: ACL; 2016. p. 2124-33.

26. Ji G, Liu K, He S, Zhao J. Distant supervision for relation extraction with sentence-level attention and entity descriptions. National Conference on Artificial Intelligence. San Francisco: AAAl; 2017. p. 3060-66.

27. Zhou P, Shi W, Tian J, Qi Z, Li B, Hao H, Xu B. Attention-based bidirectional long short-term memory networks for relation classification. Meeting of the Association for Computational Linguistics. Berlin: ACL; 2016. p. 207-12.

28. Kumar A, Irsoy O, Ondruska P, lyyer M, Bradbury J, Gulrajani I, Zhong V, Paulus R, Socher R. Ask me anything: Dynamic memory networks for natural language processing. International Conference on Machine Learning. New York; 2016. p. 1378-87.

29. Mikolov T, Chen K, Corrado G, Dean J. Efficient estimation of word representations in vector space. International Conference on Learning Representations. Scottsdale; 2013.

30. Dai AM, Olah C, Le QV. Document embedding with paragraph vectors. 2015. arXiv preprint arXiv:1507.07998.

31. Palangi H, Li D, Shen Y, Gao J, He X, Chen J, Song X, Ward R. Deep sentence embedding using long short-term memory networks: Analysis and application to information retrieval. IEEE/ACM Trans Audio Speech Lang Process. 2016;24(4):694-707.

32. Wang P, Xu B, Xu J, Tian G, Liu C-L, Hao H. Semantic expansion using word embedding clustering and convolutional neural network for improving short text classification. Neurocomputing. 2016;174(PB):806-14.

33. Shi Y, Yang Y, Liu Y. Word embedding representation with synthetic position and context information for relation extraction. 2018 IEEE International Conference on Big Knowledge (ICBK). Singapore: IEEE; 2018. p. 106-12.

34. Bengio $Y$, Simard $P$, Frasconi P. Learning long-term dependencies with gradient descent is difficult. IEEE Trans Neural Networks. 1994;5(2):157-66.

35. Graves A. Long short-term memory. Neural Comput. 1997;9(8):1735-80.

36. Mnih V, Heess N, Graves A. Recurrent models of visual attention. Advances in neural information processing systems; 2014. p. 2204-12.

37. Luong T, Pham H, Manning CD. Effective approaches to attention-based neural machine translation. Empirical Methods in Natural Language Processing. Lisbon: ACL; 2015. p. 1412-21.

38. Tran NK, Niedereée C. Multihop attention networks for question answer matching. International ACM SIGIR Conference on Research and Development in Information Retrieval. Michigan: ACM; 2018. p. 325-34.

39. Gong Y, Bowman SR. Ruminating reader: Reasoning with gated multi-hop attention. 2017. arXiv preprint arXiv:1704.07415.

40. Ao CW, Lee HY. Query-by-example spoken term detection using attention-based multi-hop networks. International Conference on Acoustics, Speech, and Signal Processing. Calgary: IEEE; 2017. p. 6264-68.

41. Alimova I, Tutubalina E. Automated detection of adverse drug reactions from social media posts with machine learning. International Conference on Analysis of Images. Cham: Springer; 2017. p. 3-15.

42. Liu S, Tang B, Chen Q, Wang X. Drug-drug interaction extraction via convolutional neural networks. Comput Math Methods Med. 2016;2016: 6918381.

43. Quan C, Hua L, Sun X, Bai W. Multichannel convolutional neural network for biological relation extraction. BioMed Res Int. 2016;2016:1-10.

44. Sahu SK, Anand A. Drug-drug interaction extraction from biomedical texts using long short-term memory network. J Biomed Inform. 2018;86:15-24.

45. Kang N, Singh B, Bui C, Afzal Z, Mulligen EMV, Kors JA. Knowledge-based extraction of adverse drug events from biomedical text. BMC Bioinformatics. 2014;15(1):1-8.

46. Sarker A, Gonzalez G. Portable automatic text classification for adverse drug reaction detection via multi-corpus training. J Biomed Inform. 2015;53:196-207.

47. Huynh T, He Y, Willis A, Rüger S. Adverse drug reaction classification with deep neural networks. International Conference on Computational Linguistics. Osaka: Coling; 2016, pp. 877-87.
48. Song X, Petrak J, Roberts A. A deep neural network sentence level classification method with context information. Empirical Methods in Natural Language Processing. Brussels: ACL; 2018, pp. 900-904.

49. Vaswani A, Shazeer N, Parmar N, Uszkoreit J, Jones L, Gomez AN, Kaiser $L$, Polosukhin I. Attention is all you need. Advances in neural information processing systems. 2017, pp.5998-6008.

\section{Publisher's Note}

Springer Nature remains neutral with regard to jurisdictional claims in published maps and institutional affiliations.
Ready to submit your research? Choose BMC and benefit from:

- fast, convenient online submission

- thorough peer review by experienced researchers in your field

- rapid publication on acceptance

- support for research data, including large and complex data types

- gold Open Access which fosters wider collaboration and increased citations

- maximum visibility for your research: over $100 \mathrm{M}$ website views per year

At BMC, research is always in progress.

Learn more biomedcentral.com/submissions 